\title{
Casimir Physics and Applications
}

\author{
Kimball Milton ${ }^{1, *,+}$ (D) and Iver Brevik $2, *,+(\mathbb{C}$ \\ 1 Homer L. Dodge Department of Physics and Astronomy, University of Oklahoma, Norman, OK 73019, USA \\ 2 Department of Energy and Process Engineering, Norwegian University of Science and Technology, \\ NO-7491 Trondheim, Norway \\ * $\quad$ Correspondence: kmilton@ou.edu (K.M.); iver.h.brevik@ntnu.no (I.B.); Tel.: +1-405-325-7060 (K.M.) \\ + These authors contributed equally to this work.
}

Received: 31 January 2019; Accepted: 31 January 2019; Published: 11 February 2019

Casimir physics encompasses all phenomena that are due to quantum field fluctuations in nontrivial backgrounds, which might be gravitational, curved space, electromagnetic (background fields or dielectric materials). It grew out of Casimir's remarkable discovery that parallel uncharged conducting plates separated by vacuum feel a force of attraction because of zero-point fluctuations in the vacuum [1] and of his work with Polder showing that neutral polarizable atoms experience retarded interactions due to quantum fluctuations [2]. The field took off when Boyer, surprisingly, found that a perfectly conducting spherical shell experienced a repulsive stress [3].

Sucn investigations continue to the present day, and applications are beginning to appear. This special issue reflects some of the theoretical concerns that are being addressed. Bordag and Pirozhenko consider a model of the photon field interacting with two slabs of matter, both represented by scalar fields [4]. Milton and Brevik re-examine models, generalizing Boyer's, in which the speed of light is the same both inside and outside a sphere [5]. Deng et al. examine what happens when the medium between the Casimir plates is anisotropic [6]. When the medium is non-uniform, represented by an external scalar potential, Fulling, Settlemyre, and Milton propose a renormalization scheme to deal with the divergences that result [7]. Fermi and Pizzocchero look at the effect on the vacuum expectation value of the stress tensor due to a point $\delta$-function singularity [8]. The implicit role of dissipation in describing the Casimir energy is explored by Guérout, Ingold, Lambrecht and Reynaud [9]. A gluing formula for calculating Casimir energies in very general piston geometries is the subject of the work by Kirsten and Lee [10].

These representative works display the vitality of the field of Casimir physics. Theorists are trying to extend Casimir's simple configuration to more realistic and elaborate situations. In the process they are discovering new problems in quantum field theory and resolving them in interesting ways that will have implications in experimental science and in applications.

Author Contributions: Both authors contributed equally to this work.

Funding: U.S. National Science Foundation, grant number 1707511; Norwegian Research Council, grant number 250346.

Conflicts of Interest: The authors declare no conflictof interest.

\section{References}

1. Casimir, H.B.G. On the Attraction Between Two Perfectly Conducting Plates. Kon. Ned. Akad. Wetensch. Proc. 1948, 51, 793.

2. Casimir, H.B.G.; Polder, D. The Influence of retardation on the London-van der Waals forces. Phys. Rev. 1948, 73, 360. [CrossRef]

3. Boyer, T.M. Quantum electromagnetic zero point energy of a conducting spherical shell and the Casimir model for a charged particle. Phys. Rev. 1968, 174, 1764. [CrossRef] 
4. Bordag, M.; Pirozhenko, I.G. Dispersion Forces Between Fields Confined to Half Spaces. Symmetry 2018, 10, 74. [CrossRef]

5. Milton, K.A.; Brevik, I. Casimir Energies for Isorefractive or Diaphanous Balls. Symmetry 2018, 10, 68. [CrossRef]

6. Deng, G.; Pei, L.; Hu, N.; Liu, Y.; Zhu, J.-R. The Impact of the Anisotropy of the Media between Parallel Plates on the Casimir Force. Symmetry 2018, 10, 61. [CrossRef]

7. Fulling, S.A.; Settlemyre, T.E.; Milton, K.A. Renormalization for a Scalar Field in an External Scalar Potential. Symmetry 2018, 10, 54. [CrossRef]

8. Fermi, D.; Pizzocchero, L. Local Casimir Effect for a Scalar Field in Presence of a Point Impurity. Symmetry 2018, 10, 38. [CrossRef]

9. Guérout, R.; Ingold, G.-L.; Lambrecht, A.; Reynaud, S. Accounting for Dissipation in the Scattering Approach to the Casimir Energy. Symmetry 2018, 10, 37. [CrossRef]

10. Kirsten, K.; Lee, Y. Gluing Formula for Casimir Energies. Symmetry 2018, 10, 31. [CrossRef]

(C) 2019 by the authors. Licensee MDPI, Basel, Switzerland. This article is an open access article distributed under the terms and conditions of the Creative Commons Attribution (CC BY) license (http:/ / creativecommons.org/licenses/by/4.0/). 